\title{
$X$-Point Effect on Plasma Blob Dynamics
}

\author{
F. Avino, A. Fasoli, I. Furno, P. Ricci, and C. Theiler \\ Ecole Polytechnique Fédérale de Lausanne (EPFL), Swiss Plasma Center (SPC), CH-1015 Lausanne, Switzerland
}

(Received 17 July 2015; published 9 March 2016)

\begin{abstract}
Plasma blob dynamics on the high-field side in the proximity of a magnetic field null ( $X$ point) is investigated in TORPEX. A significant acceleration of the blobs towards the $X$ point is observed. Close to the $X$ point the blobs break apart. The $\mathbf{E} \times \mathbf{B}$ drifts associated with the blobs are measured, isolating the background drift component from the fluctuating contribution of the blob internal potential dipole. The time evolution of the latter is consistent with the fast blob dynamics. An analytical model based on charge conservation is derived for the potential dipole, including ion polarization, diamagnetic, and parallel currents. In the vicinity of the $X$ point, a crucial role in determining the blob motion is played by the decrease of the poloidal magnetic field intensity. This variation increases the connection length that short circuits the potential dipole of the blob. Good quantitative agreement is found between the model and the experimental data in the initial accelerating phase of the blob dynamics.
\end{abstract}

DOI: 10.1103/PhysRevLett.116.105001

Magnetic field nulls ( $X$ points) are ubiquitous in space and laboratory plasmas [1-3]. The transport of particles and heat across them is of utmost importance for a variety of systems [4]. For example, much effort has been devoted by the fusion community in the past decades to investigate diverted magnetic geometries [5], which allow channeling through an $X$ point a significant fraction of the exhaust power to material surfaces. Cross-field drifts associated with the steady-state and turbulence-induced electric fields play an important role for plasma transport in the $X$-point region, as indicated by numerical and experimental studies $[6,7]$. However, experimental investigations close to $X$ points are difficult, limiting the progress in the understanding of the $X$-point dynamics and simulationexperiment comparisons. In fusion plasmas, the diagnostic accessibility is challenged by the high power flux so that the generation and propagation of intermittent plasma blobs in the vicinity of an $X$ point is largely unexplored [8-10].

In this Letter, we present the first spatial and temporaldependent in situ measurements of turbulence-generated plasma blob dynamics around the $X$-point region. The blob motion towards the $X$ point is tracked and analyzed, showing an acceleration in the initial phase that can be directly linked to the background radial flow and to the measured blob electric potential dipole. The acceleration of the blob towards the $X$ point can be quantitatively described by an analytical model that includes the dominant perpendicular and parallel current contributions. In particular, a crucial role is played by the geometrical parameter $L_{\|}$, expressing the length of the current path parallel to the magnetic field, along which the blob potential dipole is short-circuited.

The measurements are performed on the Toroidal Plasma Experiment (TORPEX) [11] with diverted magnetic geometries. Tokamaklike configurations, such as first-order ( $X$ point) or second-order (snowflake) null points, are now accessible on TORPEX with the recently installed toroidal conductor system [12]. This allows generating a rotational transform and magnetic shear [13]. The toroidal conductor is positioned at $r=(0 \pm 0.50) \mathrm{cm}, z=(16.50 \pm 0.50) \mathrm{cm}$, as indicated by the gray circle in Fig. 1. A current of about $I_{\mathrm{TC}} \simeq 640 \mathrm{~A}$ is driven in it to obtain a poloidal magnetic field. This field is superimposed to a dominant toroidal field component and a small vertical field component, both produced by external coils. The resulting magnetic configuration includes a first-order null on the low-field side (LFS), as depicted in Figs. 1(a) and 1(b) by white arrows. By setting the toroidal field to $B_{T} \simeq 0.076 \mathrm{~T}$ at $r=0 \mathrm{~cm}$, the electron cyclotron resonance layer is located at $r_{\mathrm{EC}} \simeq-14 \mathrm{~cm}$. An injected microwave power of $300 \mathrm{~W}$ results in the generation of highly reproducible, magnetized hydrogen plasmas in the region of quasivertical field lines outside the last-closed flux surface at the high-field side (HFS). Typical parameters are density $n \leq 4 \times 10^{16} \mathrm{~m}^{-3}$, electron temperature $T_{e} \leq 5 \mathrm{eV}$, ion temperature $T_{i} \ll T_{e}$, and $\beta \ll 1$, where $\beta$ is the ratio of thermal to magnetic pressure. This setup allows us to investigate plasmas in which blobs originating on the HFS propagate radially outward, exploring the $X$-point region, similarly to recent experiments in fusion plasmas, where plasma turbulence on the HFS and in the private flux region is observed $[14,15]$. The two main diagnostics are the hexagonal turbulence imaging probe (HEXTIP) [16], a 2D array of 85 fixed Langmuir probes (LPs) separated by $3.5 \mathrm{~cm}$ and covering with an hexagonal mapping the whole poloidal cross section, and a two-dimensional array of eight LPs (2DSSLP), which can be moved radially and poloidally on a shot-by-shot basis. Both systems have an acquisition frequency of $250 \mathrm{kHz}$.

Two-dimensional HEXTIP time-averaged profiles of ion saturation current density $J_{\text {sat }}$ are shown in Fig. 1(a). The black, blue, and cyan contours refer, respectively, to $80 \%$, 

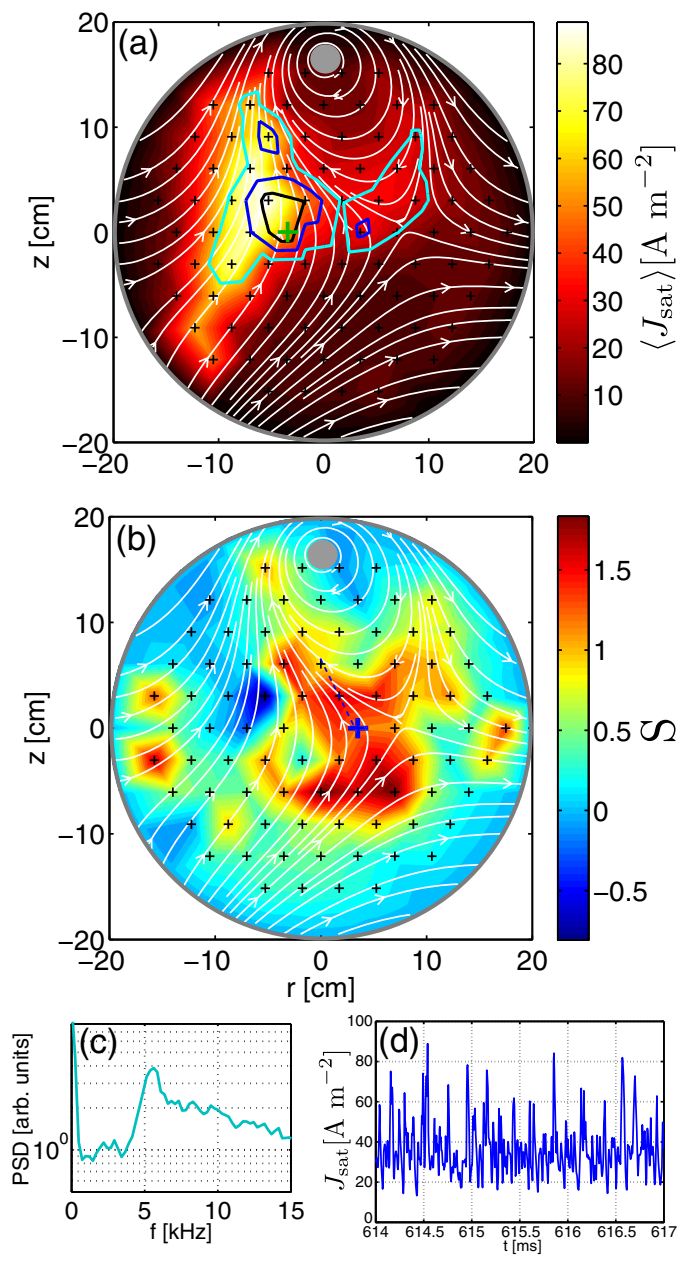

FIG. 1. Two-dimensional time average (a) and skewness (b) of HEXTIP $J_{\text {sat }}$ time traces across the TORPEX cross section. The black, blue, and cyan contours correspond to the $J_{\text {sat }}$ standard deviation iso lines at $80 \%, 60 \%$, and $40 \%$ of the measured maximum value. The PSD of the probe indicated with the green cross at $[r \simeq-3.5, z \simeq 0] \mathrm{cm}$ is shown in (c), indicating a dominant frequency at $\approx 5 \mathrm{kHz}$. The toroidal conductor is indicated with a gray circle on the top of the cross section, the white lines reproduce the calculated magnetic field lines, while the black crosses correspond to the positions of the HEXTIP LPs. In (d), the time trace of the HEXTIP probe indicated with a blue cross is reported.

$60 \%$, and $40 \%$ of the measured maximum value of $J_{\text {sat }}$ standard deviation. These indicate the high fluctuation region in which a quasicoherent mode is identified with a dominant frequency $\approx 5 \mathrm{kHz}$, as shown by the power spectral density (PSD) in Fig. 1(c) for the probe indicated with a green cross. The profile of the signal skewness in Fig. 1(b) indicates the presence of blobs in the $X$-point region, in which a detailed analysis is performed. In Fig. 1(d) we show the time trace of the LP indicated by the blue cross in Fig. 1(b), which displays a large level of intermittency associated with blobs.

Two-dimensional scans with 2DSSLP are performed over the $X$-point region. By applying a sweeping voltage to the LPs, the background plasma potential profiles are calculated from the measured $I-V$ curves as $V_{\mathrm{pl}}=$ $\mu T_{e} / e+V_{\mathrm{fl}}$, with $\mu \simeq 3.1$ [17]. The results are shown in Fig. 2(a). Time-resolved measurements of ion saturation current and floating potential $V_{\mathrm{fl}}$ provide information on the blob motion. These data are conditionally sampled and averaged [18] around the blob detection time $(\tau=0 \mu \mathrm{s})$ over a time window of $100 \mu \mathrm{s}$, determined using as a trigger the HEXTIP LP located at $[r \simeq 7, z \simeq 0] \mathrm{cm}$. An amplitude threshold corresponding to 4 times the standard deviation of the $J_{\text {sat }}$ signal is chosen for the structure detection. This analysis reveals that blobs originate in the region where a quasicoherent mode is measured, similar to studies in simpler geometries on TORPEX $[19,20]$. Once generated, the blobs propagate radially outward, moving
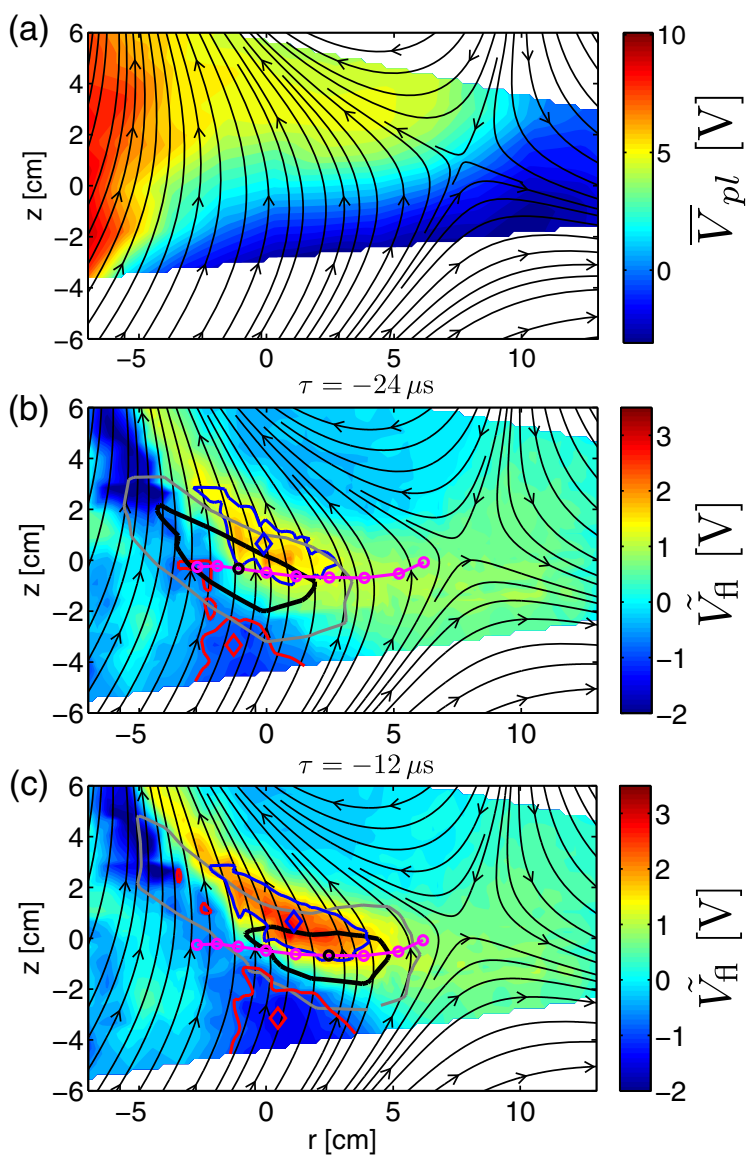

FIG. 2. (a) Two-dimensional background plasma potential profiles. (b),(c) Fluctuating floating potential $\tilde{V}_{\mathrm{fl}}$ at $\tau=$ $-24 \mu \mathrm{s}$ and $\tau=-12 \mu \mathrm{s}$ obtained from conditional average sampled data of the 2DSSLP, using as trigger the HEXTIP probe at $[r \simeq 7, z \simeq 0] \mathrm{cm}$. The blue and red contours indicate the floating potential values at $60 \%$ of the positive and negative peaks, with the weighted centers shown by the colored diamonds. The gray and black lines correspond to the $\tilde{J}_{\text {sat }}$ contours at $20 \%$ and $60 \%$ of the maximum value. The magenta curve indicates the blob trajectory, with in black the instantaneous center of mass position. 
across the $X$ point. Figures 2(b) and 2(c) show snapshots of the conditional average sampling of the measured fluctuating floating potential at $\tau=-24 \mu \mathrm{s}$ and $\tau=-12 \mu \mathrm{s}$. This reveals a dipolar potential structure associated with the blob. The two peaks of the dipole are highlighted by their $60 \%$ contour lines (blue and red) and their weighted centers (diamonds). The blob radial speed is obtained in the time window between -28 and $20 \mu \mathrm{s}$, estimating its position at each time step determined from the center of mass of the structure calculated on the interpolated data inside the $60 \%$ $\tilde{J}_{\text {sat }}$ contour. The obtained values are shown in Fig. 3 by the blue curve, while the shaded region gives an estimate of the uncertainty on the calculated speed, determined by applying the same procedure to the $40 \%$ and $80 \% \tilde{J}_{\text {sat }}$ contours. We note that after $\tau=-12 \mu$ s the conditionally averaged sampled (CAS) blob slows down, possibly because it breaks apart. This speculation is consistent with the reduction in amplitude of the CAS blob. The blob crosses the $X$ point in the time window between $\tau=4 \mu \mathrm{s}$ and $\tau=8 \mu \mathrm{s}$.

To obtain quantitative insight into the mechanisms determining the blob speed, the $\mathbf{E}_{\text {tot }} \times \mathbf{B}$ drift is evaluated by decomposing the total electric field into two main contributions:

$$
\mathbf{E}_{\text {tot }}=\overline{\mathbf{E}}_{\mathrm{bg}}+\tilde{\mathbf{E}}_{\mathrm{dip}} .
$$

$\overline{\mathbf{E}}_{\mathrm{bg}}=-\boldsymbol{\nabla} \bar{V}_{\mathrm{pl}}$ is the electric field resulting from the gradient of the steady-state plasma potential shown in Fig. 2(a). $\tilde{\mathbf{E}}_{\text {dip }}$ is the electric field originating from the blob floating potential dipole $\tilde{V}_{\mathrm{fl}}$. Both are calculated by averaging the gradients over the values inside the $60 \%$

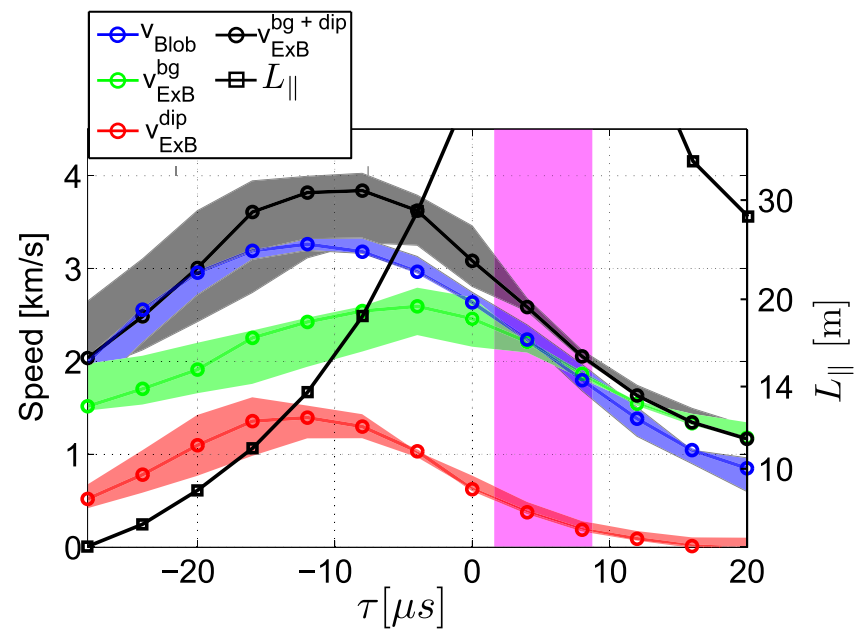

FIG. 3. In blue, experimental blob speed obtained from the blob displacement. In black circles, total blob speed calculated as the sum of the drift associated to the background plasma potential gradients (in green) and that originating from the blob potential dipole (in red). The value of the parallel connection length at the positions of the blob center of mass is indicated with black squares. The shaded area in magenta corresponds to the time when the blob is crossing the $X$ point, between $\tau=4 \mu$ s and $\tau=8 \mu \mathrm{s}$.
$\tilde{J}_{\text {sat }}$ contour. We neglect in a first approximation the fluctuating temperature contribution to the plasma potential dipole. The two corresponding contributions to the blob speed, $v_{E \times B}^{\mathrm{bg}}=\bar{E}_{\mathrm{bg}} / B$ and $v_{E \times B}^{\mathrm{dip}}=\tilde{E}_{\mathrm{dip}} / B$, are indicated in Fig. 3 by the green and the red curve. The shaded regions provide an estimate of the uncertainty on the calculated curves, obtained by averaging the electric fields inside the $40 \%$ and $80 \% \quad \tilde{J}_{\text {sat }}$ contours. We find that both terms contribute significantly to the total $\mathbf{E} \times \mathbf{B}$ velocity of the blob, $v_{E \times B}^{\mathrm{bg}+\text { dip }}$, shown in black in Fig. 3. The latter agrees well with the measured velocity of the blob, $v_{\text {blob }}$. We note that the velocity associated with the blob dipole plays a crucial role in the first $16 \mu \mathrm{s}$, contributing with $\approx 0.8 \mathrm{~km} / \mathrm{s}$ to the radial velocity increase of the blob.

To explain the time evolution of the blob dipole, the magnetic field shear along the blob trajectory is considered in the following way. The vertical magnetic field in the region where the blob detaches from the original mode, namely at $[r \simeq-2, z \simeq 0] \mathrm{cm}$, is approximately $3 \mathrm{G}$. This value and the associated magnetic geometry allows us to infer the toroidally uniform feature of the fluctuations $\left(k_{\|} \neq 0, k_{\mathrm{tor}}=0\right)$, referring to the resistive interchange regime previously studied in the simple magnetized torus configuration $[21,22]$. This is supported by the vertical distance covered by a field line after a toroidal turn $(\sim 2 \mathrm{~cm})$, which is comparable to the blob vertical size at $60 \%$ of its $\tilde{J}_{\text {sat }}$ maximum, indicated in Fig. 2(b). These observations suggest that the detached blob is not a field aligned structure connected to the walls, but that it closes on itself [19]. This is compatible with the measurements of the coherence between LPs positioned at different toroidal angles. Under these assumptions, the magnetic field provides a path $L_{\|}$for the parallel currents to short circuit the vertical potential dipole. A schematic of this situation is shown in Fig. 4, in which the vertical, radial, and toroidal coordinates are indicated with $\hat{\mathbf{z}}, \hat{\mathbf{r}}$, and $\hat{\mathbf{y}}$. The values of $L_{\|}$explored by the blob are indicated in Fig. 3.

As the blob approaches the $X$ point, the parallel connection length $L_{\|}$increases. This effect can be taken into account by evaluating the blob speed $v_{b}$ starting from charge conservation:

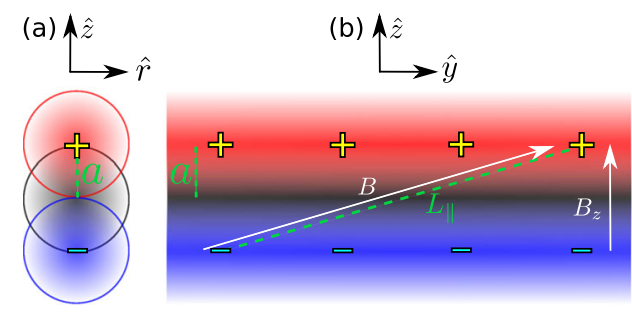

FIG. 4. Schematic of blob density profile (black) with the associated potential dipole (red and blue) on the poloidal cross section (a) and along the toroidal direction (b), with the parallel connection length $L_{\|}$and the blob size $a$ indicated. 


$$
\boldsymbol{\nabla} \cdot \mathbf{J}=\boldsymbol{\nabla}_{\perp} \cdot \mathbf{J}_{\perp}+\boldsymbol{\nabla}_{\|} J_{\|}=0,
$$

in a way similar to the previously reported scaling laws $[10,23]$. The main difference is the replacement of the parallel current to the sheath with the parallel current short circuiting the blob dipole inside the plasma. We focus on this term using the hypothesis of axisymmetry $\left(k_{\text {tor }}=k_{y}=0\right)$ by writing the parallel gradient as

$$
\nabla_{\|}=b_{z} \frac{\partial}{\partial z}+b_{y} \frac{\partial}{\partial y}=\frac{B_{z}}{B} \frac{\partial}{\partial z}, \quad \text { with } \quad b_{z}=\frac{B_{z}}{B},
$$

where $B_{z}$ is the poloidal magnetic field and $B$ the total magnetic field. It follows that

$$
\nabla_{\|} J_{\|}=\frac{B_{z}}{B} \frac{\partial J_{\|}}{\partial z}
$$

The parallel electric field can now be evaluated to obtain the parallel current $J_{\|}=\sigma E_{\|}$, with $\sigma$ the effective plasma conductivity:

$$
E_{\|}=\mathbf{E} \cdot \mathbf{b}=E_{y} b_{y}+E_{z} b_{z}=-\frac{\partial \tilde{\phi}}{\partial z} \frac{B_{z}}{B} .
$$

Here, $\tilde{\phi}$ is the fluctuating blob potential. We therefore obtain

$$
\nabla_{\|} J_{\|}=-\sigma \frac{B_{z}}{B} \frac{\partial}{\partial z}\left(\frac{B_{z}}{B} \frac{\partial \tilde{\phi}}{\partial z}\right) \approx-\sigma\left(\frac{B_{z}}{B}\right)^{2} \frac{\partial^{2} \tilde{\phi}}{\partial z^{2}} .
$$

We note that, due to the toroidal symmetry, the parallel dynamics is only given by the perpendicular gradients. The perpendicular current density contribution of Eq. (2) is very similar to that in Ref. [23], where we consider the ion polarization and the electron diamagnetic currents. We finally get

$$
-\sigma\left(\frac{B_{z}}{B}\right)^{2} \frac{\partial^{2} \tilde{\phi}}{\partial z^{2}}+\frac{2 c_{s}^{2} m_{i}}{R B} \frac{\partial n}{\partial z}-\frac{m_{i} n}{B^{2}} \frac{d}{d t}\left(\nabla_{\perp}^{2} \tilde{\phi}\right)=0,
$$

where $c_{s}=\sqrt{T_{e} / m_{i}}$ is the ion sound speed and $R$ the major radius. We can proceed with the same estimates performed in Ref. [23]:

$$
\begin{array}{rlrl}
\frac{\partial^{2} \tilde{\phi}}{\partial z^{2}} & \sim-\frac{2 \tilde{\phi}}{(2 a)^{2}}, & & \nabla_{\perp}^{2} \tilde{\phi} \sim-\frac{\tilde{\phi}}{a^{2}}, \\
\frac{\partial n}{\partial z} \sim-\frac{\delta n}{a}, & \frac{d}{d t} \sim \frac{\sqrt{2} c_{s}}{\sqrt{R a}} .
\end{array}
$$

In addition, we write

$$
\frac{B_{z}}{B} \sim \frac{2 a}{L_{\|}},
$$

where $L_{\|}$is the parallel connection length along the magnetic field between the two peaks of the blob dipole, sketched in Fig. 4. The following expression is obtained:

$$
\frac{2 c_{s}^{2} m_{i}}{R B} \frac{\delta n}{a}=\sigma \frac{2 \tilde{\phi}}{L_{\|}^{2}}+\frac{m_{i} n}{B^{2}} \frac{\sqrt{2} c_{s}}{\sqrt{R a}} \frac{\tilde{\phi}}{a^{2}} .
$$

The blob speed can be estimated from $\tilde{\phi} \sim B v_{b} a$ and $\sigma=C n$, where $C$ indicates the proportionality coefficient between the plasma conductivity and the plasma density:

$v_{b}=\frac{\delta n}{n} \sqrt{\frac{2 a}{R}} c_{s}\left(\frac{1}{1+A / L_{\|}^{2}}\right), \quad A=\frac{C B^{2} a^{5 / 2} \sqrt{2 R}}{m_{i} c_{s}}$.

For $L_{\|} \rightarrow \infty$, the inertial scaling $v_{b}=\delta n / n \sqrt{2 a / R} c_{s}$ is retrieved [10]. Since, experimentally, the blob size does not perfectly coincide with half of the distance between the dipole peaks entering in Eq. (9), an intermediate value $a=$ $1.5 \mathrm{~cm}$ between the blob size contour at $60 \%$ and the dipole width is chosen. Deriving a scaling law with a different blob size and distance between the dipole peaks does not introduce significant changes in the results. Referring to the temperature $T_{e} \simeq 5 \mathrm{eV}$ measured in the mode region, we estimate $c_{s} \simeq 2.2 \times 10^{4} \mathrm{~m} / \mathrm{s}$. The velocity $v_{b}$ can then be evaluated at each time step considering $\delta n / n$ and $B$ (hence, $\left.L_{\|}\right)$the only position-dependent variables. We note that $L_{\|}$is the most significant parameter in the spatial variation of the estimated speed, increasing in the range [7-14] $\mathrm{m}$, while $\delta n / n$ is almost constant for most of the trajectory. The coefficient $C$ is determined by adjusting the plasma conductivity to make the analytically estimated speed coincide with the experimental value at $\tau=-28 \mu \mathrm{s}$. We note that we find $C \simeq 2 \times 10^{-14} \mathrm{C}^{2} \mathrm{~kg}^{-1} \mathrm{~s}$, which is 3 times less the value we would obtain by estimating the conductivity as $\sigma=n e^{2} / m_{e} \nu_{e H}$, with $\nu_{e H}=n_{n} \sigma_{e H} \sqrt{T_{e} / m_{e}} \simeq 5 \times 10^{5} \mathrm{~s}^{-1}$, considering a neutral density $n_{n} \simeq 5 \times 10^{18} \mathrm{~m}^{-3}$, an electron-neutral collision cross section $\sigma_{e H}=10^{-19} \mathrm{~m}^{2}$.

The resulting values are indicated by the blue curve in Fig. 5 for the time window between -28 and $-4 \mu \mathrm{s}$. Good

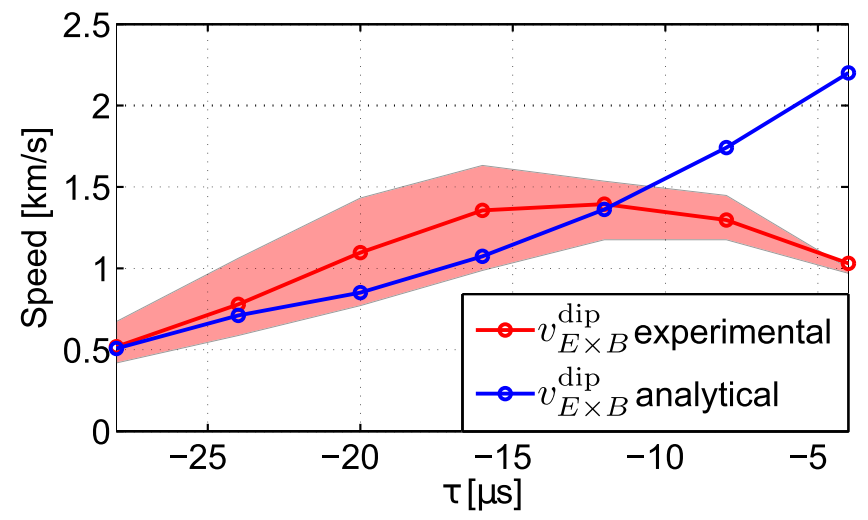

FIG. 5. In blue, blob scaling speed evaluated during the acceleration phase with the introduced analytical model. In red, the measured contribution of the fluctuating blob dipole at the corresponding times. 
agreement is found during the accelerating phase between the model and the experimentally measured contribution of the fluctuating potential dipole (red curve). Using instead the estimated value of $C$ would reduce the calculated initial velocity by $0.3 \mathrm{~km} / \mathrm{s}$, while keeping the acceleration almost unchanged. We note that for times larger than $-12 \mu \mathrm{s}$, the model stops to work, which is consistent with the already discussed indications that the blobs start to break apart.

In summary, the dynamics of plasma blobs in the proximity of a single null $X$ point has been investigated with unprecedented detail. The experimental conditions imply the presence of blobs that are not field aligned, nor connected to any material surface. The blob radial motion is determined by the $\mathbf{E} \times \mathbf{B}$ flows associated with the background electric field and with the blob potential dipole. Their variation along the blob trajectory is consistent with the measured acceleration. An analytical model based on charge conservation has been developed to explain the potential dipole variation, which is largely responsible for the acceleration of the blob as it approaches the $X$ point. In this model, a crucial role is played by the field line geometry, providing a parallel path for the short circuit of the blob dipole, whose importance varies as the blob moves towards the $X$ point. A good quantitative agreement is found between the measured blob velocities and the blob speed scaling evaluated at the same time steps during the initial accelerating phase. The analyzed data show that the blob dynamics is strongly influenced by the presence of an $X$ point. We disentangled the role of background and fluctuating drifts, showing that both play an important role in determining the blob motion in the considered $X$-point configuration, and therefore they are crucial quantities to estimate in fusion devices.

This work was supported in part by the Swiss National Science Foundation. This work has been carried out within the framework of the EUROfusion Consortium and has received funding from the European Union's Horizon 2020 research and innovation program under Grant Agreement No. 633053. The views and opinions expressed herein do not necessarily reflect those of the European Commission. Support from the EUROfusion Grants No. EF-WP14-FRF-EPFL/Theiler and No. EF-WP15-ENR-EPFL-05 is gratefully acknowledged. The authors wish to acknowledge very helpful discussions with the SPC colleagues A. Bovet, F. Nespoli, and N. Vianello, and the support of the SPC technical team. *fabio.avino@epfl.ch

[1] W. Fundamenski, V. Naulin, T. Neukirch, O. E. Garcia, and J. Juul Rasmussen, Plasma Phys. Controlled Fusion 49, R43 (2007).

[2] M. Yamada, R. Kulsrud, and H. Ji, Rev. Mod. Phys. 82, 603 (2010).

[3] G. Fiksel, W. Fox, A. Bhattacharjee, D. H. Barnak, P.-Y. Chang, K. Germaschewski, S.X. Hu, and P. M. Nilson, Phys. Rev. Lett. 113, 105003 (2014).

[4] P. Hill, F. Hariri, and M. Ottaviani, Phys. Plasmas 22, 042308 (2015).

[5] C. S. Pitcher, and P. C. Stangeby, Plasma Phys. Controlled Fusion 39, 779 (1997).

[6] J. A. Boedo, M. J. Schaffer, R. Maingi, and C. J. Lasnier, Phys. Plasmas 7, 1075 (2000).

[7] M. J. Schaffer, J. A. Boedo, R. A. Moyer, T. N. Carlstrom, and J. G. Watkins, J. Nucl. Mater. 290-293, 530 (2001).

[8] A. Kirk, B. Koch, R. Scannell, H. R. Wilson, G. Counsell, J. Dowling, A. Herrmann, R. Martin, and M. Walsh, Phys. Rev. Lett. 96, 185001 (2006).

[9] S. I. Krasheninnikov, D. A. D'Ippolito, and J. R. Myra, J. Plasma Phys. 74, 679 (2008).

[10] D. A. D'Ippolito, J. R. Myra, and S. J. Zweben, Phys. Plasmas 18, 060501 (2011).

[11] S. H. Müller, A. Fasoli, B. Labit, M. McGrath, M. Podestà, and F. M. Poli, Phys. Rev. Lett. 93, 165003 (2004).

[12] F. Avino, A. Fasoli, and I. Furno, Rev. Sci. Instrum. 85, 033506 (2014).

[13] F. Avino, A. Fasoli, I. Furno, S. Jolliet, and P. Ricci, Phys. Plasmas 21, 122115 (2014).

[14] S. Potzel, M. Wischmeier, M. Bernert, R. Dux, H. W. Müller, and A. Scarabosio, J. Nucl. Mater. 438, S285 (2013).

[15] J. R. Harrison, G. M. Fishpool, and B. D. Dudson, J. Nucl. Mater. 463, 757 (2015).

[16] S. H. Müller, A. Fasoli, B. Labit, M. McGrath, O. Pisaturo, G. Plyushchev, M. Podestà, and F. M. Poli, Phys. Plasmas 12, 090906 (2005).

[17] M. Podestà, A. Fasoli, B. Labit, M. McGrath, S. H. Müller, and F. M. Poli, Plasma Phys. Controlled Fusion 47, 1989 (2005).

[18] H. Johnsen, H. L. Pécseli, and J. Trulsen, Phys. Fluids 30, 2239 (1987).

[19] S. H. Muller, C. Theiler, A. Fasoli, I. Furno, B. Labit, G. R. Tynan, M. Xu, Z. Yan, and J. H. Yu, Plasma Phys. Controlled Fusion 51, 055020 (2009).

[20] I. Furno et al., Phys. Rev. Lett. 100, 055004 (2008).

[21] F. M. Poli, P. Ricci, A. Fasoli, and M. Podestà, Phys. Plasmas 15, 032104 (2008).

[22] P. Ricci and B. N. Rogers, Phys. Rev. Lett. 104, 145001 (2010).

[23] C. Theiler, I. Furno, P. Ricci, A. Fasoli, B. Labit, S. H. Müller, and G. Plyushchev, Phys. Rev. Lett. 103, 065001 (2009). 